\title{
Exact Harmonic Coefficients for a Magnetic Ring Head
}

\author{
David T. Wilton, Barry K. Middleton, Member, IEEE, and Mustafa M. Aziz
}

\begin{abstract}
The magnetic field of a ring head has been analyzed by Westmijze, using a conformal mapping, and by Fan, using Fourier techniques. Here these methods are reexamined and combined to give, for the first time, an explicit analytic expression for the harmonic coefficients in the Fan solution.
\end{abstract}

Index Terms - Heads, frequency response, magnetic fields, mathematics, recording.

\section{INTRODUCTION}

$\mathbf{O}$ NE of the most popular magnetic recording head geometries is the familiar ring head. Two approaches to the analysis of the field of such a head are conformal mapping and Fourier analysis, each of which leads to analytic results although neither is entirely satisfactory in this respect. Westmijze [1] first provided a conformal mapping solution but the mapping requires numerical inversion so that the final result is not fully analytic. Fan's [2] Fourier analysis does provide an explicit result but this is in the form of an infinite series whose coefficients have been determined by solving a large (theoretically infinite) system of linear equations.

Here, each of these methods is examined again and by combining them, two techniques arise which lead to formulas for the coefficients in the Fan solution, thus avoiding the need to solve large systems of equations. The first, motivated by a remark of Mallinson [3], gives an explicit formula for the coefficients, while the second gives a procedure for determining each coefficient in terms of the preceding ones.

The motivation for publication of these results is three-fold. First, the fact that the Fan coefficients, which have previously been computed by a number of authors [2], [4]-[6] and measured experimentally [7] with varying degrees of success, have (after nearly 40 years!) now been derived precisely, is intrinsically interesting and will hopefully make the Fan solution more widely accessible. Second, the first technique described should have wider applicability to other head geometries where a Fourier (Fan-type) solution is known. And third, publicizing successful techniques of combining apparently unrelated methods may encourage similar approaches in other problems.

Manuscript received September 21, 1998; revised January 14, 1999.

D. T. Wilton is with the School of Mathematics and Statistics and the Centre for Research in Information Storage Technology, University of Plymouth, Plymouth, Devon PL4 8AA, U.K. (e-mail: d.wilton@plymouth.ac.uk).

B. K. Middleton and M. M. Aziz are with the Division of Electrical Engineering, University of Manchester, Manchester M13 9PL, U.K.

Publisher Item Identifier S 0018-9464(99)02788-0.

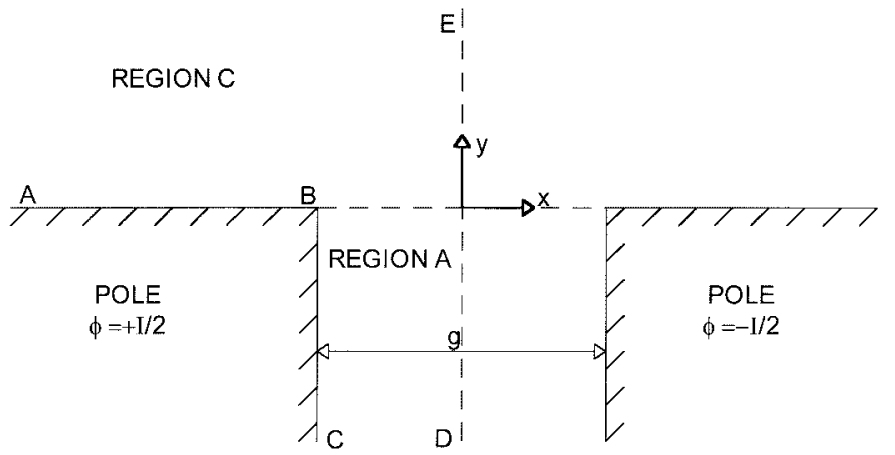

Fig. 1. Ring head model in the $z$ plane.

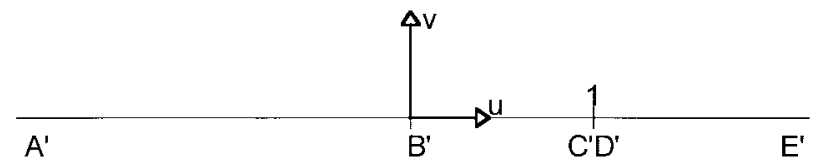

Fig. 2. The complex $w$ plane.

\section{REVIEW OF METHODS}

\section{A. Conformal Mapping}

First the Westmijze solution is briefly reviewed. The idealized model geometry of a ring head is shown in Fig. 1 where two semi-infinite pole pieces are at magnetic potentials $\pm I / 2$ and separated by a gap of total length $g$. The Schwarz-Christoffel transformation

$$
\frac{d z}{d w}=S \frac{\sqrt{w}}{w-1}
$$

maps the boundary ABCDE (for half a ring head) in the $z$ plane to the real axis in the $w$ plane with the corresponding points $\mathrm{A}^{\prime}, \mathrm{B}^{\prime}, \mathrm{C}^{\prime}, \mathrm{D}^{\prime}, \mathrm{E}^{\prime}$ shown in Fig. 2. Integration of (1) gives

$$
z=S\left[2 \sqrt{w}+\ln \left(\frac{\sqrt{w}-1}{\sqrt{w}+1}\right)\right]+z_{0}
$$

and ensuring that $\mathrm{CD} \rightarrow \mathrm{C}^{\prime} \mathrm{D}^{\prime}$ and $\mathrm{B} \rightarrow \mathrm{B}^{\prime}$ leads to $S=$ $i g / 2 \pi$ and $z_{0}=0$.

The magnetostatic potential function in the upper half $w$ plane which takes the value $I / 2$ on the real $w$ axis for $-\infty<\operatorname{Re}(w)<1$ and zero for $\operatorname{Re}(w) \geq 1$ is $\operatorname{Im}[F(w)]$ where

$$
F(w)=\frac{I}{2 \pi} \ln (w-1) .
$$


By standard theory [8], the components of the magnetic field $\boldsymbol{H}=-\nabla \phi$ in the $z$ plane are given by

$$
H_{y}+i H_{x}=-\frac{d F(w)}{d w} \frac{d w}{d z}
$$

producing here

$$
H_{x}-i H_{y}=\frac{I}{g \sqrt{w}} .
$$

To determine the field components at any point $z=x+i y$ in the $z$ plane, the corresponding point $w$ needed in (5) may be found from (2), using, say, the Newton-Raphson numerical iterative method.

Two results, derived in [1], are particularly useful here. Westmijze computed fields along the boundary line $\mathrm{A}^{\prime} \mathrm{B}^{\prime} \mathrm{C}^{\prime} \mathrm{D}^{\prime} \mathrm{E}^{\prime}$ where $w$ is real and hence $\sqrt{w}$ is easily evaluated. In particular along $\mathrm{DE}, \sqrt{w}=\sqrt{u}=I /\left[g H_{x}(0, y)\right]$ giving

$$
x=0, \quad y=\frac{g}{\pi}\left[U^{-1}+\frac{1}{2} \ln \left(\frac{1-U}{1+U}\right)\right]
$$

where $U=H_{x}(0, y) / H_{0}$ and $H_{0}=I / g$, the deep gap field.

Westmijze also calculated the flux through the coil, using the reciprocity principle, in terms of a "gap loss" function

$$
\begin{aligned}
S(\tau)= & \operatorname{Im}\left[\frac{1}{2 \pi} \int_{-\infty}^{1} \frac{1}{1-w}\right. \\
& \left.\cdot e^{(2 \tau / \pi)[\sqrt{w}+(1 / 2) \ln ((\sqrt{w}-1) /(\sqrt{w}+1))]} d w\right] .
\end{aligned}
$$

If

$$
\hat{H}_{x}(k, y)=\int_{-\infty}^{\infty} H_{x}(x, y) e^{-i k x} d x
$$

is the Fourier transform of the horizontal magnetic field, $\hat{H}_{x}(k, y)$ and $S(\tau)$ are related by

$$
\hat{H}_{x}(k, y)=I e^{-k y} S(k g / 2) .
$$

\section{B. Fourier Solution}

Now consider Fan's Fourier solution. In the gap $(-g / 2 \leq$ $x \leq g / 2,-\infty<y \leq 0$, region A) the general solution of Laplace's equation taking the correct potentials on the pole pieces is

$$
\phi_{A}(x, y)=-\frac{I x}{g}-\sum_{n=1}^{\infty} A_{n} \sin \left(\frac{2 n \pi x}{g}\right) e^{2 n \pi y / g}
$$

while above the poles $(-\infty<x<\infty, 0 \leq y<\infty$, region $\mathrm{C})$

$$
\phi_{C}(x, y)=\int_{0}^{\infty} C(\sigma) \sin \left(\frac{2 \sigma x}{g}\right) e^{-2 \sigma y / g} d \sigma .
$$

Matching the potential along $y=0$ gives

$$
C(\sigma)=-\frac{I}{\pi} \frac{\sin (\sigma)}{\sigma^{2}}-\sum_{n=1}^{\infty} A_{n} 2 n(-1)^{n} \frac{\sin (\sigma)}{\sigma^{2}-(n \pi)^{2}}
$$

and matching the vertical field at $y=0$ in the gap leads to an infinite set of linear equations which, until now, needed to be solved to give the normalized harmonic coefficients $A_{m}^{\prime}=A_{m} /(I / 2)$.

\section{EXACT HARMONIC COEFFICIENTS}

\section{A. Technique 1}

The first method presented follows similar work by Mallinson [3] for the "thin" gap head of Westmijze [1] which resulted in an explicit form for the appropriate harmonic coefficients as $2 J_{0}(n \pi) /(n \pi)$. From (11), $C(\sigma)$ is effectively the Fourier transform of the magnetic potential in the head-face plane $(y=0)$, since for $y \geq 0$

$$
\hat{\phi}(k, y)=-i \frac{\pi g}{2} C(k g / 2) e^{-k y}
$$

giving

$$
\hat{H}_{x}(k, y)=-\frac{k \pi g}{2} C(k g / 2) e^{-k y} .
$$

Now put $\sigma=m \pi$ in (12), where $m$ is an integer, to give

$$
C(m \pi)=-\frac{A_{m}}{\pi}
$$

and then from (14) and (15)

$$
A_{m}=\frac{1}{m \pi} \hat{H}_{x}(2 m \pi / g, y) e^{2 m \pi y / g} .
$$

As observed in [3], the harmonic coefficients are simply multiples of sampled values of the spectral response function $\hat{H}_{x}(k, y) e^{k y}=-i k \hat{\phi}(k, 0)$ at $k=2 m \pi / g$.

This result establishes the link with the conformal mapping solution via (9) giving the normalized coefficients as

$$
A_{m}^{\prime}=\frac{2}{m \pi} S(m \pi) \text {. }
$$

It remains to evaluate the integral (7) when $\tau=m \pi$. If the range of integration is split, the substitution $w=s^{2}$ for $0 \leq w \leq 1$ shows that the integrand is totally real there and hence gives no contribution to the result, while the substitution $w=-s^{2}$ for $-\infty<w \leq 0$ leads to

$$
S(m \pi)=\operatorname{Im}\left[\frac{1}{2 \pi} \int_{-\infty}^{\infty} \frac{s}{1+s^{2}}\left(\frac{i s-1}{i s+1}\right)^{m} e^{2 m i s} d s\right] .
$$

This integral may be evaluated by complex contour integration on closing the contour in the upper half complex $s$ plane and using the method of residues. There is only one pole of the integrand, at $s=i$, and setting $t=s-i$ leads to $S(m \pi)=K_{m} e^{-2 m}$ where $K_{m}$ is the coefficient of $t^{m}$ in $(t+i)(t+2 i)^{m-1} e^{2 m i t}$.

Now

$$
\begin{aligned}
K_{m}= & \sum_{n=0}^{m-1}\left[\left(\begin{array}{c}
m-1 \\
n
\end{array}\right)(2 i)^{m-1-n}\right] \\
& \cdot\left[\frac{-(2 m i)^{m-n-1}(m+n)}{(m-n) !}\right]
\end{aligned}
$$

where the term in the first bracket is the coefficient of $t^{n}$ in the binomial expansion of $(t+2 i)^{m-1}$ and the term in the 
TABLE I

Exact Harmonic COEFFicients For a Ring HeAd

\begin{tabular}{|c|c|c|}
\hline$m$ & $A_{m}^{\prime}$ & $A_{m}^{\prime}$ (correct to 8 d.p.) \\
\hline 1 & $-\frac{2 e^{-2}}{\pi}$ & -0.08615712 \\
\hline 2 & $\frac{5 e^{-4}}{\pi}$ & 0.02915024 \\
\hline 3 & $-\frac{58 e^{-6}}{3 \pi}$ & -0.01525422 \\
\hline 4 & $\frac{539 e^{-8}}{6 \pi}$ & 0.00959250 \\
\hline 5 & $-\frac{6934 e^{-10}}{15 \pi}$ & -0.00668033 \\
\hline 6 & $\frac{38081 e^{-12}}{15 \pi}$ & 0.00496516 \\
\hline 7 & $-\frac{918970 e^{-14}}{63 \pi}$ & -0.00386090 \\
\hline 8 & $\frac{109167851 e^{-16}}{1260 \pi}$ & 0.00310358 \\
\hline 9 & $-\frac{166282598 e^{-18}}{315 \pi}$ & -0.00255909 \\
\hline 10 & $\frac{9303339907 e^{-20}}{2835 \pi}$ & 0.00215301 \\
\hline
\end{tabular}

second bracket is the coefficient of $t^{m-n}$ in the Taylor series expansion of $(t+i) e^{2 m i t}$. This simplifies to

$$
K_{m}=\sum_{n=0}^{m-1}\left(\begin{array}{c}
m-1 \\
n
\end{array}\right) \frac{m+n}{(m-n) !}(-1)^{m-n}(4 m)^{m-n-1}
$$

Hence the normalized harmonic coefficients are

$$
A_{m}^{\prime}=\frac{2 e^{-2 m}}{m \pi} K_{m}
$$

The first ten values are shown in Table I. The values given in [6] are seen to be correct to the 6 d.p. quoted there.

\section{B. Technique 2}

The second technique which enables exact evaluation of the harmonic coefficients uses the Westmijze result (6) which may be rearranged as

$$
U=\tanh \left(U^{-1}-y \pi / g\right) .
$$

Using an exponential series for tanh [9] gives

$$
U=1+2 \sum_{n=1}^{\infty}(-1)^{n} e^{-2 n U^{-1}} e^{2 n \pi y / g} .
$$

Another expression for $U$ is found from the Fourier representation (10) as

$$
U=1+\sum_{n=1}^{\infty} A_{n}^{\prime} n \pi e^{2 n \pi y / g}
$$

The idea now is to match the coefficients of powers of $\varepsilon=e^{2 \pi y / g}$ in each of (23) and (24) but first (24) must be substituted into the exponential term on the right-hand side of (23) since (23) is implicit in $U$. If $\alpha_{n}=A_{n}^{\prime} n \pi$, (24) becomes

$$
U=1+\sum_{n=1}^{\infty} \alpha_{n} \varepsilon^{n}
$$

and then (23) is

$$
U=1+2 \sum_{n=1}^{\infty}(-1)^{n} e^{-2 n\left(1+\Sigma_{n=1}^{\infty} \alpha_{n} \varepsilon^{n}\right)^{-1}} \varepsilon^{n} .
$$

Expanding $U^{-1}$ in (26) as a power series in $\varepsilon$ valid for $\left|\sum_{n=1}^{\infty} \alpha_{n} \varepsilon^{n}\right|<1$, which is satisfied for $y$ sufficiently negative, expressing $e^{-2 n U^{-1}}$ as a Taylor series and then equating coefficients of $\varepsilon^{n}$ in (25) and (26) leads to

$$
\begin{array}{ll}
n=1: & \alpha_{1}=-2 e^{-2} \\
n=2: & \alpha_{2}=-4 e^{-2} \alpha_{1}+2 e^{-4}=10 e^{-4} \\
n=3: & \alpha_{3}=-4 e^{-2} \alpha_{2}+8 e^{-4} \alpha_{1}-2 e^{-6}=-58 e^{-6}
\end{array}
$$

etc. Each $\alpha_{n}$ is given in terms of previous ones but it has not proved possible to establish a general formula.

The computer algebra package Mathematica [10] has been used to produce further values which are entirely consistent with the coefficients $A_{n}^{\prime}$ in Table I and provides a valuable independent check on their correctness.

\section{CONCLUSIONS}

A fresh look at two long-established techniques for determining the magnetic field of a ring head has given further insight into this problem. In particular an explicit formula for the harmonic coefficients of Fan's solution has been derived. Also the relationship between such coefficients and the spectral response/gap loss function, observed in [3], has been reinforced. Such a relationship holds for any head geometry where a Fourier representation of the form (11) is valid.

\section{ACKNOWLEDGMENT}

The authors would like to thank Dr. P. W. James for helpful discussions on this problem.

\section{REFERENCES}

[1] W. K. Westmijze, "Studies on magnetic recording," Philips Res. Rep., vol. 8, pp. 161-183, 1953.

[2] G. J. Y. Fan, "A study of the playback process of a magnetic ring head," IBM J. Res. Develop., vol. 5, pp. 321-325, Oct. 1961.

[3] J. C. Mallinson, "Westmijze's 'thin' gap head revisited," IEEE Trans. Magn., vol. 26, pp. 3140-3154, Nov. 1990.

[4] A. W. Baird, "An evaluation and approximation of the Fan equations describing magnetic fields near recording heads," IEEE Trans. Magn., vol. 16, pp. 1350-1352, Sept. 1980.

[5] H. L. Huang and H. Y. Deng, "Comparison of ring head and SPT head write fields," IEEE Trans. Magn., vol. 22, pp. 1305-1309, Sept. 1986.

[6] D. T. Wilton, "Comparison of ring and pole head magnetic fields," IEEE Trans. Magn., vol. 26, pp. 1229-1231, May 1990.

[7] A. K. Dinnis, B. K. Middleton, and J. J. Miles, "Characteristics of magnetic recording heads," Meas. Sci. Technol., vol. 3, pp. 362-365, Apr. 1992.

[8] K. J. Binns and P. J. Lawrensen, Analysis and Computation of Electromagnetic and Magnetic Field Problems. Oxford, U.K.: Pergamon, 1973. 
[9] I. S. Gradshteyn and I. M. Ryzhik, Tables of Integrals, Series and Products. New York: Academic, 1993

[10] S. Wolfram, The Mathematica Book. Cambridge, U.K.: Wolfram Media; Cambridge Univ. Press, 1996.

David T. Wilton received the B.A. degree in mathematics from the University of York, U.K., in 1969 and the D.Phil. degree in numerical analysis from the University of Oxford, U.K., in 1974.

He spent three years at the University of Dundee, Scotland, and then three years with the Ministry of Defence working on dynamic fluid-structure interaction problems in underwater acoustics. Since 1978 he has been lecturing mathematics at the University of Plymouth, U.K. From 1987-1989 he was at the City Polytechnic of Hong Kong. His main research interests are in numerical analysis and applied mathematics in the areas of electromagnetics and acoustics.
Barry K. Middleton (M'96) was born in Solihull, Warwickshire, U.K. He received the B.Sc. degree in physics from the University of Sheffield, U.K.

$\mathrm{He}$ has worked at International Computers and Tabulators (Engineering) Ltd, Stevenage, Hertfordshire, U.K., on magnetic storage and logic systems, at the University of Salford, U.K., on magnetic materials for recording and bubble domain applications, and at Manchester Polytechnic, U.K., on magnetic recording. He is now at the University of Manchester, U.K., where he is Head of the Electronic and Information Storage Systems Research Group and is working on magnetic recording in tape and disk systems.

Mustafa M. Aziz was born in 1972. He received the B.Eng. degree in electrical engineering from Salford University, U.K., in 1995. He is currently working towards the Ph.D. degree at the University of Manchester, U.K.

His work has involved the signal and noise properties of thin film recording media. 